\title{
Fonction de répartition des averses en Tunisie
}

\section{Functions portraying the distribution of showers in Tunisia}

\author{
C. Thirriot \\ Institut National Polytechnique de Toulouse
}

\author{
K. Maallel et M. Triki
}

Ecole Nationale d'Ingénieurs de Tunis

\section{Introduction}

L'Office National d'Assainissement de Tunisie (ONAS) réalise actuellement un vaste plan d'aménagement des systèmes urbains de drainage des eaux pluviales.

Sous l'incitation de M. Khrouf, Directeur des Etudes à l'ONAS, des études ont été lancées à l'ENIT ayant pour objet une meilleure connaissance du régime des averses en Tunisie à partir des observations dont disposent la Météorologie Nationale Tunisienne et la Direction des Ressources en Eau et Sol du Ministère de l'Agriculture (DRES).

Cette communication donne une vue des premiers résultats obtenus.

Inventaire des données disponibles

\section{Quelques définitions}

\section{Probabilité - fréquence - Période de retour}

Soit $N$ le nombre total d'averses de la population.

Soit $n(I)$ le nombre d'averses d'intensité inférieure ou égale à $I$ pour la durée fixée $t_{0}$.

$F\left(I, t_{0}\right)=n(I) / N$ représente le pourcentage en nombre des averses d'intensité $I$ pour une durée $t_{0}$. A partir des valeurs de ces pourcentages, on va pouvoir construire le graphe empirique des fréquences cumulées $F\left(I, t_{0}\right)$ ou fonction de répartition empirique de probabilité.

Nous avons choisi de classer les averses par ordre croissant. En fait, ce qui va nous intéresser, ce sont les intensités très fortes. Aussi nous aurons à considérer la probabilité d'apparition de pluies d'intensité $I^{\prime}$ supérieure à $I$ dont la valeur est complémentaire de la proba- bilité d'apparition de pluies d'intensité $I^{\prime \prime}$ inférieure à $I$.

$$
\operatorname{Prob}\left(I^{\prime}>I\right)=1-\operatorname{Prob}\left(I^{\prime \prime}<I\right)
$$

Nous désignerons par $F^{\prime}$ cette probabilité :

$$
F^{\prime}=1-F
$$

Reste à définir la période de retour.

Supposons que la population d'averses examinées ait été observée sur une durée totale de $D$ années.

La taille de la population étant $N$, on suppose qu'en moyenne le nombre d'averses est uniformément réparti sur la durée $D$.

La probabilité de l'évènement annuel est donc :

$$
F_{1}^{\prime}=\frac{D}{N}
$$

A cet évènement annuel, sur le graphe de la fonction de répartition correspond une intensité $I$ de pluie annuelle de durée $t_{0}$.

Le temps de retour (ou période de retour) de l'événement annuel est évidemment l'année.

Par analogie avec cette notion concrète et familière, on définit plus généralement un temps (ou période) de retour $T$ compté en années auquel correspond une fréquence cumulée ou probabilité $F^{\prime}=D / N T$ et à cette fréquence $F^{\prime}(T)$ correspond une intensité $I\left(t_{0}, T\right)$ dépendant de la durée de pluie fixée à priori.

\section{Etudes antérieures}

\section{Les différents travaux réalisés en Tunisie}

La première étude des averses en Tunisie a été faite par M. Bonenfant en 1935 sur la station Tunis-Manoubia, sur la période allant de 1901 à 1934. Dans cette étude, 
M. Bonenfant s'est limité à l'élaboration des courbes empiriques.

En 1953, M. de Montmarin a travaillé sur les données de pluviographes de Tunis. Ces pluviographes étaient hebdomadaires, du type à siphon; ils ne permettaient pas d'apprécier les intensités sur les durées de référence inférieures à $20 \mathrm{~min}$. Les intensités proposées par M. de Motmarin étaient de la forme :

$$
I=\frac{a}{b+t}
$$

En 1964, Monsieur Cormary a établi des courbes intensité-durée-fréquence pour 46 stations de la Tunisie. Partant de paramètres établis à partir d'autres données climatologiques et grâce à des diagrammes américains, M. Cormary estime les intensités maximales moyennes de durée 1 heure et 24 heures et de période de retour 2 ans. Il généralise la loi $I=f(t T)$ à partir des données journalières $I(24 \mathrm{~h}, T)$ et $I(24 \mathrm{~h}, 2$ ans $)$ suivant la règle de proportionnalité ci-dessous :

$$
I(t, T)=\frac{I(24 \mathrm{~h}, T)}{I(24 \mathrm{~h}, 2 \text { ans })} \cdot I(t, 2 \text { ans })
$$

Ces courbes constituaient le seul document officiel publié par la Direction des Ponts et Chaussées.

En 1971, M. Boussabah reprit l'étude de M. de Montmarin en lui ajoutant le dépouillement des vingt dernières années :1950-1970. Dans cette étude M. Boussabah a bien mis en évidence la différence des résultats obtenus à partir des observations de 1909-1950 et celles de 1950-1970; pour une même période de retour et une même durée de référence, les vingt dernières années donnaient des intensités de $80 \%$ à $100 \%$ plus fortes que la première période. Trois facteurs sont à l'origine de cette différence :

- le changement de pluviographe (hebdomadaire à siphon avant 1950, journalier à augets basculants après 1950);

- le changement du mode de dépouillement (à l'aide d'une grille transparente avant 1950, découpage systématique toutes les cinq minutes après 1950);

- le changement de période d'observation.

L'étude la plus récente est celle de M. Saidi sur la station de Tunis-Carthage pour les dépouillements des années 1950-1970. Cette étude a le mérite de présenter une nouvelle méthode d'ajustement permettant de mieux décrire les évènements de faible période de retour (un à dix mois).

\section{Comparaison des résultats}

Nous représentons sur le graphe (Fig. 1), pour les averses décennales et annuelles, les courbes $I=f(t)$ des différentes méthodes : de Montmarin, Boussabah, Cormary, Saidi et Djougar. En analysant ces graphes, nous remarquons que l'écart maximal entre les intensités des différentes études va du simple au double.

\section{Conclusion de l'étude bibliographique}

$\mathrm{Vu}$ l'importance des courbes $I-D-F$ dans le calcul des ouvrages d'assainissement et la discordance

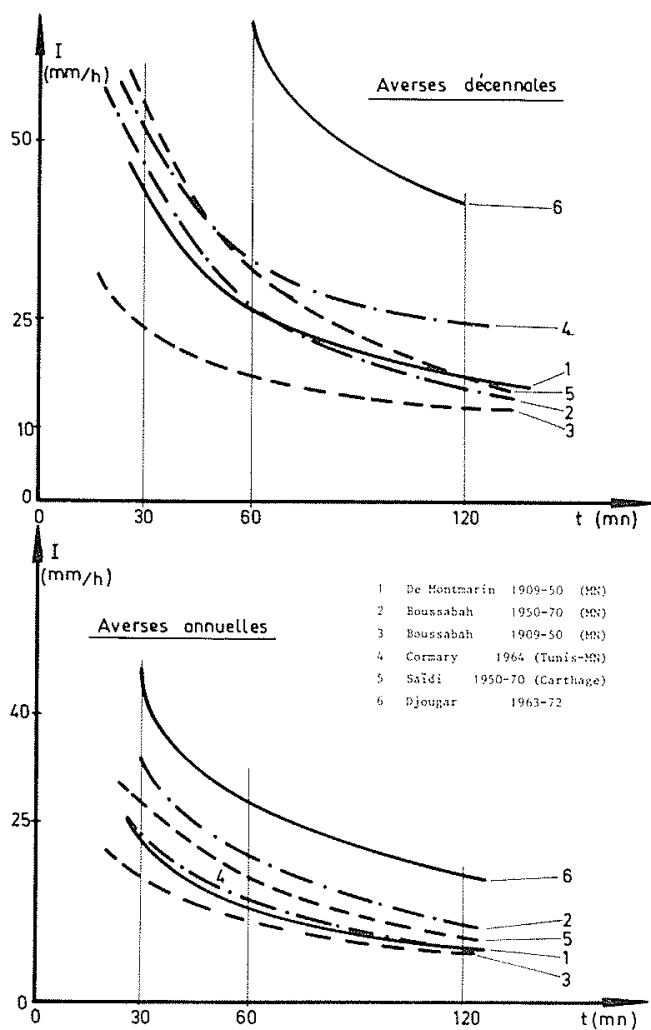

Figure 1

des résultats des différentes études hydrologiques, nous nous sommes proposés de reprendre l'étude en tenant compte des dernières années d'observations.

\section{Sources d'information}

Les données pluviométriques se réduisent aux dépouillements des pluviogrammes de 1950 à 1970 pour les stations météorologiques de Tunis-Carthage et de Tunis-Manoubia et de dix ans pour huit autres stations à l'intérieur de la Tunisie (Fig. 2).

Des pluviogrammes existent pour le reste des stations mais ils ne sont pas dépouillés par manque de temps et de personnel. Le dépouillement des pluviogrammes d'une année pour une station demande un minimum d'un mois de travail pour les gens de la Météorologie.

Devant ce manque de données, nous avons dépouillé manuellement les pluviogrammes des huit dernières annés pour les stations de Tunis-Manoubia et de TunisCarthage, travail qui nous a permis d'avoir ainsi vingt neuf ans d'observations pour ces deux stations.

\section{Traitement statistique}

Anamorphose de l'argument dans l'ajustement des fonctions empiriques de répartition

Pour une durée fixée $t$, l'hydrologue obtient un graphe discret de probabilités cumulées dont il cherche habituellement une représentation approchée analytique continue qui offrira deux possibilités : assurer un 


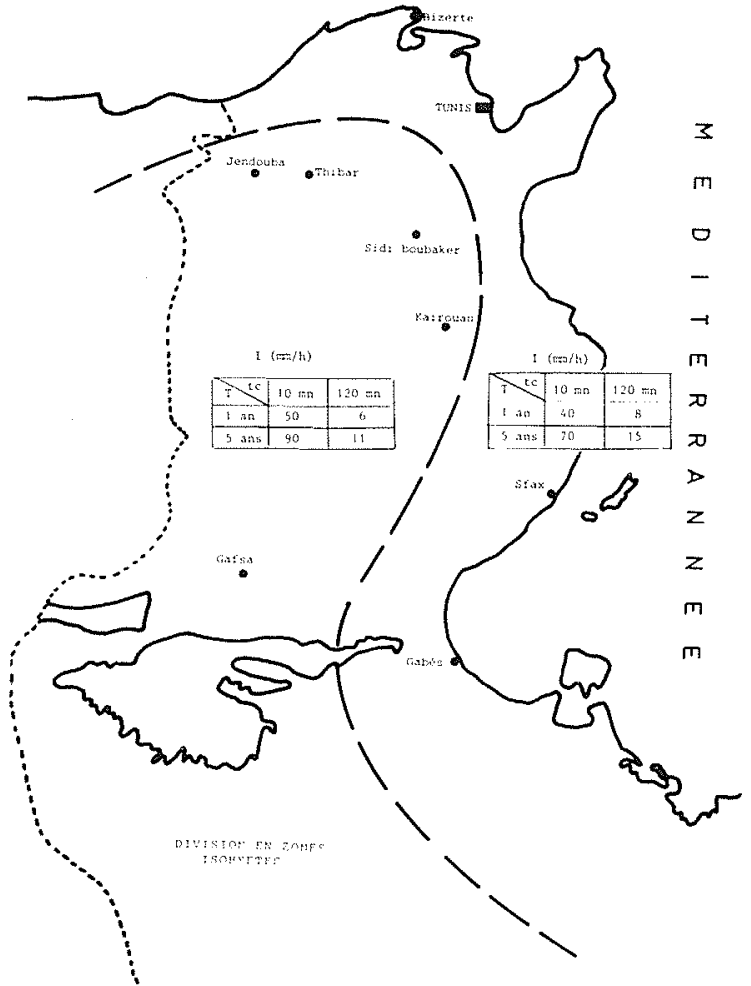

Figure 2

lissage des résultats d'observations et permettre l'extrapolation.

Dans les tentatives d'ajustement, on commence par essayer les fonctions qui ont un bien fondé statistique ou probabiliste comme la fonction erreur ou loi normale ou les fonctions dérivant de la statistique des extrêmes (Frechet, Gumbel). Mais, a priori, toute fonction variant de 0 à 1 de manière monotone peut être essayée comme approximation de la fonction empirique de répartition. Par exemple :

$$
\begin{aligned}
& F=\frac{1}{2}\left(1-\cos \pi \frac{x}{\lambda}\right) \text { pour } x \in[0, \lambda] \\
& \left.F=\frac{1}{2}\left(1-\operatorname{th} \frac{x}{\lambda}\right) x \in\right]-\infty,+\infty[
\end{aligned}
$$

Il suffit encore que la fonction de densité de probabilité ne présente qu'un macimum entre deux points où elle s'annule telle que les fonctions eulériennes $A x^{\alpha}(\lambda-x)^{\beta}$ pour $x \in[0, \lambda]$ ou $A x^{\alpha} e^{-\beta x}$ pour $x \in[0, \infty[$ (classe dont font partie les fonctions de répartition de Pearson).

La diversité du choix des fonctions d'ajustement est encore accrue par le changement de variable. Il est traditionnel de passer de la variable aléatoire physique $x$ à son logarithme Log $x$ comme c'est le cas dans l'étude des distributions de débit. Mais nous avions remarqué en examinant les résultats présentés dans sa thèse par Bernard Bobée qu'une même distribution empirique, représentée avec une échelle gaussienne en ordonnée, conduisait à une concavité de sens différent suivant qu'on utilisait $x$ ou $\log x$ comme argument. Comme dans la plupart des ajustements graphiques, on cherche à rectifier le graphe empirique, cette remarque nous avait conduit à imaginer un changement progressif sur l'argument au lieu du saut que constitue le passage de $x$ à $\log x$. Et l'étude des averses tunisiennes à servi de champ d'expérimentation à cette idée très simple.

Partant de la tradition, nous avons cherché à relier $x$ et $\log x$ grâce à l'anamorphose $x^{\alpha}$. En effet, l'origine du logarithme peut être vue sous la forme intégrale :

$$
\log x=\int_{1}^{x} \frac{d u}{u}
$$

qui n'est que le cas particulier de

$$
\phi=\int_{1}^{x} \frac{d u}{u^{1-\alpha}}
$$

pour $\alpha$ tendant vers 0 .

Quelques coups de sonde numériques montrent la bonne proximité de $\phi(x, \alpha)$ et de $\log x$ pour $\alpha$ très petit

$$
\phi(x, \alpha)=\frac{1}{\alpha}\left(x^{\alpha}-1\right)
$$

\section{Mise en ouvre automatique des ajustements}

A partir des courbes classées $I(t)$ pour chaque averse ou par lecture directe sur les enregistrements pour quelques durées $t$, on obtient les fonctions de répartition empiriques $F(I, t)$

$\mathrm{Ce}$ graphe discret empirique est l'élément de base de toute la suite du travail. Entré dans la mémoire de l'ordinateur, il va être manipulé pour en donner le meilleur ajustement analytique.

Le principe de l'ajustement s'inspire du procédé manuel; il consiste à rectifier le graphe, c'est-à-dire, à rendre le graphe anamorphosé le plus proche possible d'un graphe linéaire.

Les amorphoses sont de deux sortes :

- anamorphose de la fonction en essayant différentes lois de répartition et en prenant chaque fois la fonction réciproque $Z(F)$;

- anamorphose de la variable $Y(I)=I^{\alpha}$.

L'objectif est d'obtenir une relation approchée

$$
Z=A Y+B
$$

Les coefficients $A$ et $B$ sont déterminés automatiquement par la méthode des moindres carrés. (Fig. 3).

\section{Inversion des fonctions de répartition}

L'objectif de l'ajustement d'une loi de répartition est d'approcher au mieux une relation linéaire $Z(Y)$. Pour ce faire, il faut utiliser la fonction réciproque $Z(F)$. Bien sûr, on peut envisager d'effectuer des interpolations pour des fonctions tabulées mais nous avons préféré par souci de simplicité disposer d'une formulation explicite de la fonction. réciproque. D'où le choix des fonctions de répartition que nous avons utilisées. 

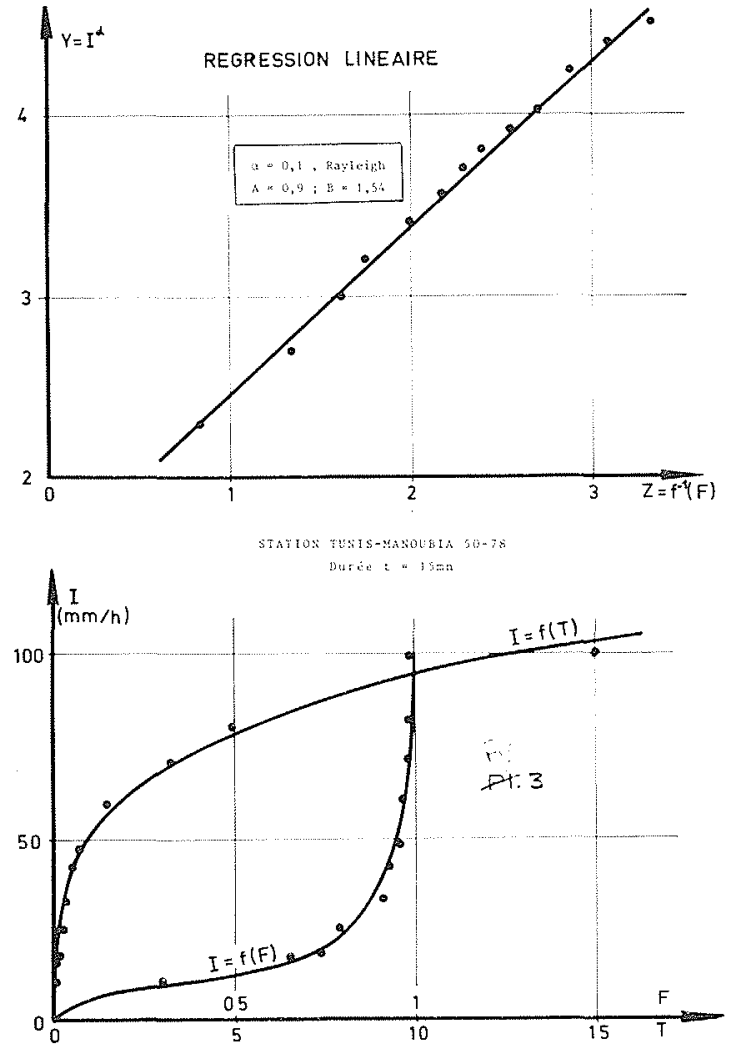

Figure 3

Pour certaines, l'inversion est immédiate.

La distribution de Rayleigh $F=1-e^{-Z^{2} / 2}$ conduit à :

$$
Z=\sqrt{-2 \log (1-F)}
$$

La fonction de Gumbel $F=e^{-e^{-Z}}$ donne :

$$
Z=-\log (-\log F)
$$

L'un de nous a proposé, lors d'étude de courbes granulométriques, d'utiliser une fonction de répartition inspirée de la fonction de Gumbel et que nous désignerons par loi IMFT

$$
\left.F=1-e^{-e^{Z}} Z \epsilon\right]-\infty,+\infty[
$$

dont la fonction réciproque est

$$
Z=\log [-\log (1-F)]
$$

Une classe importante de fonction de répartition concerne la traditionnelle loi normale pour laquelle nous utiliserons une fonction réciproque approchée assez simple élaborée par $\mathrm{C}$. Thirriot :

$$
\begin{array}{ll}
\text { si } & F<0,5 \quad Z=2,15 \frac{F-0,5}{F^{1 / 6}} \\
\text { si } & F>0,5 \quad Z=2,15 \frac{F-0,5}{(1-F)^{1 / 6}}
\end{array}
$$

\section{Critère de sélection de la classe d'ajustement}

A partir de l'ajustement on estime $Y_{i}^{\prime}\left(Z_{i}\right)$ valeur lissée correspondant à la fréquence empirique $Z_{i}$ afférente à $Y_{i}$ observé.

Les critères de qualité de l'ajustement examinés par l'ordinateur sont de trois sortes : la variance $E_{1}^{2}$, l'écart relatif moyen en $E_{2}$ et une somme pondérée $10 E_{1}+E_{2}$.

\section{Mise en ouvre automatique}

L'ensemble des opérations décrites ci-dessus est effectué automatiquement par l'ordinateur qui signale en clair sur l'imprimante le nom de la loi qui convient le mieux.

Le programme donne aussi directement le paramètre de Student pour juger de l'intervalle de confiance.

\section{Graphes intensité-durée}

Disposant des différents graphes $F\left(I, t_{i}\right)$ pour les 6 valeurs de $t_{i}$ déjà mentionnées $(5,10,20,30,60$ et $120 \mathrm{~min}$ ) pour une population donnée (c'est-à-dire une station) on va chercher les graphes $I\left(t, F_{k}\right)$ soit encore les graphes $I\left(t, T_{k}\right)$ donnant l'évolution de l'intensité de la pluie en fonction de sa durée pour une période de retour fixée.

Après différentes tentatives, nous nous en sommes tenus sagement aux formules classiques de Montana

$$
I=a_{1} t^{b_{1}}
$$

de Talbot $I=\frac{a_{2}}{b_{2}+t}$

Les coefficients $a_{j}$ et $b_{j}$ des deux formules sont calculés par régression linéaire traitée directement par l'ordinateur.

Les résultats de l'interpolation dont on peut voir un exemple sur la station de Tunis-Carthage, figure 4, ne sont pas fameux. L'écart relatif moyen est souvent supérieur à $10 \%$. Ceci tient à la rigidité du corset que constituent les formules de Montana et Talbot à deux paramètres.

La considération de la moyenne améliore un peu la proximité avec le graphe empirique. Mais alors implicitement on utilise un graphe à 4 paramètres $a_{1}, a_{2}$, $b_{1}, b_{2}$.

\section{Etude critique des résultats}

\section{Sensibilité aux données et aux hypothèses de départ}

Pour l'étude critique, nous utiliserons surtout les résultats afférents aux stations de Tunis-Manoubia et de Tunis-Carthage où les observations sont les plus nombreuses. La formules examinée est celle de Montana $I=a t^{b}$.

\section{Durée de l'intervalle de référence}

La durée de l'intervalle de référence varie entre 5 et 120 minutes pour la station de Tunis-Manoubia et 


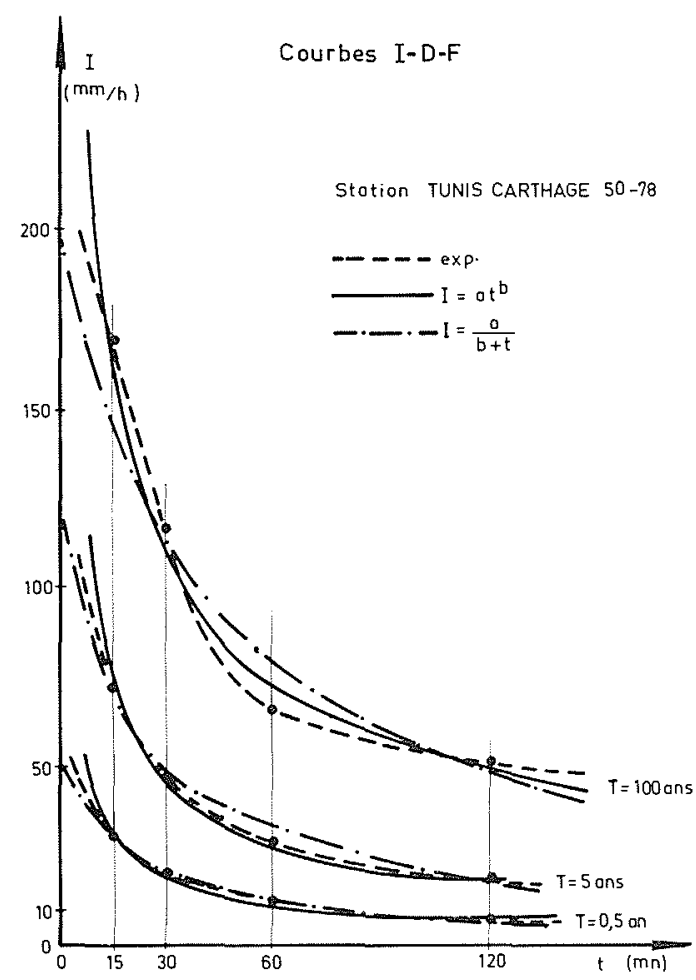

Figure 4

entre 15 et 120 minutes pour celle de Tunis-Carthage.

Les courbes $a(T) b(T)$ afférentes à $t=15$ min pour Tunis-Carthage sont bien placées entre les courbes de $10 \mathrm{~min}$ et $20 \mathrm{~min}$ de Tunis-Manoubia. D'où nous pouvons conclure que les deux stations ont les mêmes courbes $I D F$. Cette conclusion est importante vu l'hétérogénéité des épisodes pluvieux entre les deux stations distantes de dix kilomètres environ. Cette échelle est à comparer avec la dimension des cellules de pluie proposée par P. Misme et étudiée aussi par G. Bediot, V.M. Bellostas, C. Bocquillon et J.M. Masson sur les orages en région parisienne.

\section{Influence de la période d'observation}

Les données pluviométriques disponibles sont limitées à dix ans pour la plupart des stations météorologiques. Avant d'entreprendre l'étude de ces données, nous avons voulu examiner la reprćscntativité de 10 ans par rapport à l'ensemble des données d'une même station. Pour cela, nous avons divisé les observations de Tunis-Manoubia en trois échantillons de 10,10 , et 9 ans correspondant respectivement aux périodes 50-59, 60-69 et 70-78. Ces périodes concordent avec un cycle météorologique bien connu par les spécialistes, en relation directe avec les taches solaires.

L'analyse des graphes $a_{(T)}, b_{(T)}$ montre que ces courbes restent voisines les unes des autres d'où on peut admettre qu'ils appartiennent à une même population sans sortir des intervalles de confiance choisis au départ.

\section{Influence du nombre d'averses dépouillées}

a) Elimination du premier seuil

L'élimination du premier seuil réđuit le nombre total des averses dépouillées de près de $50 \%$. L'examen des résultats nous a permis de conclure que l'influence des averses de grande fréquence est petite et que les différences d'intensité qui en résultent peuvent-être admises sans sortir des intervalles de confiance.

\section{b) Etude des maximums annuels}

Nous avons réduit le nombre d'averses au nombre d'années d'observations en ne considérant que les maximums annuel d'intensité pour chaque intervalle de référence. Les coefficients $a$ et $b$ des courbes $I D F$ n'évoluent pas de la même façon en fonction de la période de retour; c'est pourquoi, on peut conclure que les maximums annuels ne sont pas représentatifs de la population de l'ensemble des averses.

\section{Conclusion}

Bien que l'influence de la durée de l'intervalle de référence soit bien nette et que dix ans d'observations soient assez représentatifs, il faut aller prudemment dans l'interprétation et le classement des résultats des autres stations. Car si les données des stations de TunisCarthage et de Tunis-Manoubia sont bien étudiée, vérifiées et parfois même corrigées, on n'est pas sûr que ce soit le cas des autres stations de l'intérieur dont les données sont encore à l'état brut.

\section{Répartition géographique des paramétres carac- téristiques}

\section{Etude des autres stations}

Dans ce qui précède, nous nous sommes intéressés à l'information météorologique concernant la station de Tunis.

Cette étude détaillée nous a permis de mettre au point la méthodologie.

L'information météorologique concernant le reste de la Tunisie est moins riche qu'à Tunis ou tout au moins, moins facilement disponible.

Cependant, nous avons pu traiter toutes les données pluviométriques disponibles à l'Institut National de la Météorologie. Le tableau suivant donne la répartition de ces données :

\begin{tabular}{|l|c|c|}
\hline \multicolumn{1}{|c|}{ Station } & Durée (ans) & Date \\
\hline Kairouan & 10 & $49-58$ \\
Bizerte & 10 & $49-58$ \\
Thibar & 10 & $54-65$ \\
Jendouba & 10 & $49-58$ \\
Sidi Bou Baker & 20 & $51-70$ \\
Sfax & 10 & $49-58$ \\
Gafsa & 10 & $49-58$ \\
Gabès & 20 & $49-58$ \\
\hline
\end{tabular}

\section{Courbes intensité-durée-fréquence définitives}

Les courbes intensité-durée-fréquence pour l'ensemble des stations avec les coefficients $a_{(T)}, b_{(T)}$ de la formule 


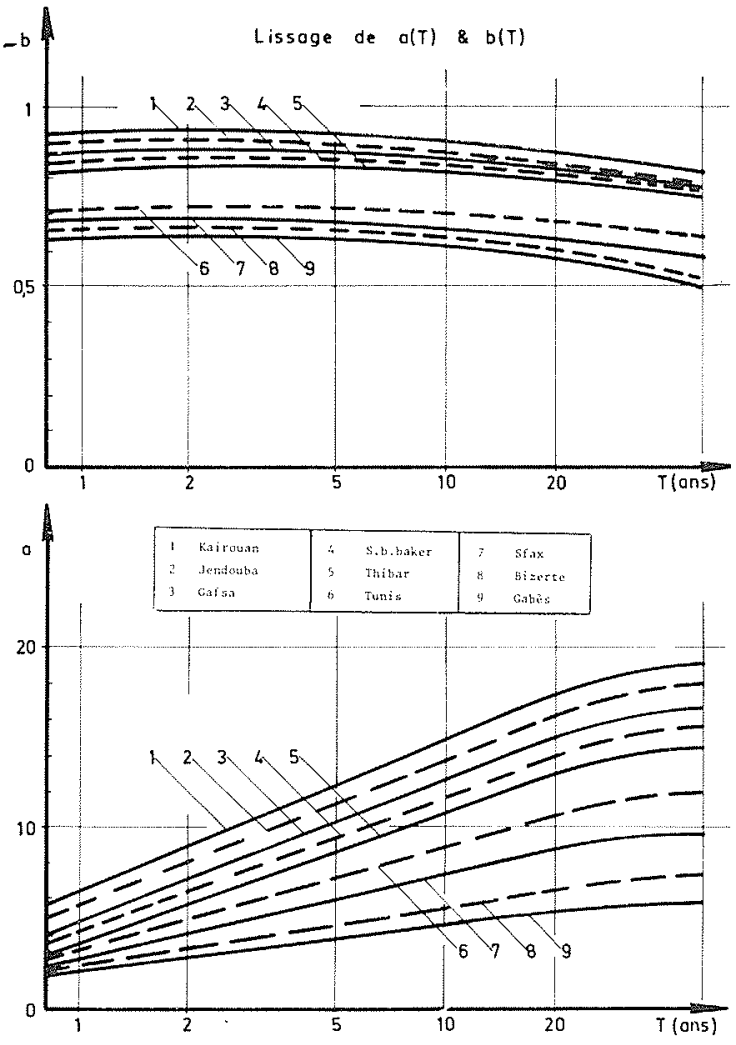

Figure 5

de Montana $I=a t^{b}$ sont donnees par un troisième programme et ont conduit aux graphes de la figure 5 .

\section{Groupement des stations}

L'examen de ces courbes a permis de grouper ces stations en deux séries : la première est caractéristique des fortes intensités de durée inférieure à une heure, la seconde des fortes intensités de durée supérieure à une heure (Fig. 2).

Les stations de la première série sont placées sur la côte; celles de la deuxième série sont à l'intérieur; d'autre part, à l'intérieur d'une même série les différences d'intensité pour une durée et une période de retour données restent à l'intérieur des intervalles de confiance fixés au départ ( $5 \%$ sur $I$ ). D'où, nous avons proposé des courbes IDF pour ces deux-régions. Les coefficients $a_{(T)}$ et $b_{(T)}$ sont déterminés à partir des coefficients de l'ensemble des stations de chaque région

La distinction du régime littoral rejoint les conclusions de G. Escourou sur les "fortes pluies de la bande côtière en Normandie".

\section{Conclusion}

Malgré la somme de travail accumulée au cours de cette étude des averses en Tunisie, il reste encore beaucoup à faire sur le plan technique et sur le plan scientifique.

La constitution d'une banque des données météorologiques qui s'enrichit au cours des années jointe à l'existence d'une bibliothèque de programmes de traitement automatique permettra la mise à jour et l'affinement des courbes intensité-durée-fréquence pour les différentes zones climatiques de Tunisie.

\section{Remerciements}

Au cours de cette étude hydrologique des averses en Tunisie, les chercheurs de l'ENIT ont bénéficié du concours de nombreux spécialistes compétents et dévoués.

Il n'est pas possible de les nommer tous mais qu'ils veuillent bien trouver ici l'assurance de notre gratitude pour leur accueil courtois et le partage cordial de leur expérience.

Bien entendu, il s'agit d'abord des cadres de l'ONAS, maître d'oeuvre de cette étude, mais aussi des ingénieurs de la DRES, de la Météorologie Nationale et des Bureaux d'études tels que la SCET, la SOTUETEC et la GIC.

Enfin, nous tenons aussi à remercier Madame M.H. Puech de l'IMFT pour sa collaboration intelligente, dévouée et efficace dans la préparation des différents rapports afférents à cette étude et Monsieur J.P. Bombaud pour le bénéfice décisif dû à son expérience dans l'élaboration des premiers programmes.

\section{Bibliographie}

BEDIOT G., BELLOSTAS J.M., BOCQUILLON C. et MASSON J.M. - Pluies orageuses en région parisienne. Numéro Spécial Précipitations et Hydrologie $\mathrm{VI}^{\mathrm{e}}$ Série, Mars et Juin 1980, $\mathrm{n}^{\circ} 20-21$, p. 281-291.

BESBES M. - L'estimation des apports aux nappes souterraines : un modèle régional d'infiltration efficace. Thèse d'Etat, Université P. et M. Curie, 1978

BOBEE B. - Contribution à l'étude des débits maximaux de crues : représentation par des distributions Pearson type III et Log-Pearson type III. Thèse de Docteur-Ingénieur, 1976.

BONENFANT. - Evaluation des réseaux d'eau pluviales en Tunisie. Note 5020097 DRES, 1935.

BOUSSABAH. - Etudes des intensités maximales d'averses à Tunis-Manoubia. Note 50 8018C DRES, 1971.

CORMARY Y. Les pluies en Tunisie (loi, intensité, durée). Note TM 152 DRES, 1964.

DESBORDES M. - Réflexions sur les méthodes de calcul des réseaux urbains d'assainissement pluvial. Thèse, Université de Montpellier, 224 p., Février 1974.

DESBORDES M. - Une étude générale de l'assainissement pluvial urbain. La Houille Blanche, 1975, n ${ }^{\circ}$ 1, p. 37-43.

DRES. - Monographie de l'Oued Miliane. Note DRES.

DRES. - Note sur les crues exceptionnelles de 1973 surl'Oued Zita à Gabès. Note DRES.

ESCOUROU G. - Les fortes pluies de la bande côtière en Normandie. Numéro Spécial Précipitations et Hydrologie, $\mathrm{VI}^{\mathrm{e}}$ Série, Mars et Juin 1980, ${ }^{\circ}$ 20-21. p. 295-298.

Instruction Technique relative aux réseaux d'assainissement des agglomérations. Imprimerie Nationale, Paris, 1978.

KALLEL. - Traitement sur ordinateur des données hydrologiques. Note TH 165, DRES.

LAHAYE J.P. - Etude des hauteurs de pluie exceptionnelles en un ou plusieurs jours consécutifs en Haute-Volta. Numéro Spécial Précipitations et Hydrologie, $\mathrm{VI}^{\mathrm{e}}$ Série, Mars et Juin $1980, n^{\circ} 20-21$, p. 191-197.

LEVIANDIER T. - Liaison spatiale entre postes pluviométriques; corrélation en fonction de la distance et du temps. Unn modèle de simulation. Numéro Spécial Précipitations et Hydrologie, VI ${ }^{\mathrm{e}}$ Série, Mars et Juin 1980, $\mathrm{n}^{\circ}$ 20-21, p. 255-261. 
MISME P. - Dimension des cellules de pluie. Numéro Spécial Précipitations et Hydrologie, VI ${ }^{\mathrm{e}}$ Série, Mars et Juin 1980 , $\mathrm{n}^{\circ}$ 20-21, p. 299-302.

MONTMARIN de. - Intensité et durée des averses enregistrées à Tunis. Note 5020100 DRES, 1953.

MONTMARIN de. - Les pluies torrentielles en Tunisie. Note TM 182 DRES.
REMENIERAS G. - Hydrologie de l'Ingénieur. Eyrolles, 1965. SAID1. - Intensité des précipitations à Tunis-Carthage. Note Météorologie Nationale.

SAIDI. - Les valeurs météorologiques extrêmes, leurs ajuste. ments, leurs durées de retour. Note Méteorologie Nationale. Seminaire d'Hydrologie Urbaine, animé par Michel Desbordes. ENIT, Tunis, Juin 1979

\section{Discussion}

Président: M. R. DARVES-BORNOZ

Le Président. - Je remercie M. le Professeur THIRRIOT de cette communication intéressante, qui appellera sans doute des questions.

Qui est le premier à poser des questions à M. THIRRIOT ?

M. LAHAYE - 1) A t-on conduit, parallèlement à ces études, des programmes de mesure d'hydrologie urbaine en Tunisie?

2) Lorsqu'on désire effectuer un ajustement de loi statistique on est amené à résoudre deux problèmes:

- choisir un type de loi adopté (Gumbel, Gauss, loi empirique ou autre).

- déterminer au mieux les paramètres numériques de la loi choisie.

Pour résoudre le premier problème, il est nécessaire d'effectuer une étude régionale car il parait anormal je crois, qu'un échantillon suive une loi donnée en un lieu et que l'on observe une loi différente à proximité. En fait, l'erreur d'échantillonnage est très importante pour des séries courtes et l'ajustement très précis, en chaque station, par des textes numériques apporte une précision illusoire.

3) Concernant ce non recouvrement des deux zones (intérieur et côte) avec les courbes isohyètes annuelles. on observe le même phénomène en Afrique Occidentale en zone soudano-sahelienne où l'on peut considérer une zone unique pour les courbes intensité-durée-fréquence alors que la pluviométrie annuelle varie de 300 à $1200 \mathrm{~mm}$.

M. THIRRIOT. - J'essaierai de répondre succinctement à M. LAHAYE.

1) Pour ce qui concerne les débits, la Direction des Etudes de l'ONAS a lancé une campagne de mesure en zone urbaine et semi-urbaine avec la coopération des organismes universitaires tunisiens (INAT, ENIT) et de l'Université de LUND en Suède. Actuellement les mesures sont effectuées avec enregistrement sur cassette.

2) En réponse à la question sur le choix de la loi statistique, il faut distinguer, d'une part, le souci d'ajustement de la loi dé répartition pour une station, afin de permettre interpolation et extrapolation pour différentes périodes de retour mais toujours pour cette station et d'autre part, la tentative d'avoir une vue générale et synthétique pour l'ensemble d'un territoire et, dans ce but, nous avons retenu la loi de Montana à causc de sa simplicité et de son emploi répandu (par excmple dans la formule de Caquot).

A propos de la précision sur les paramètres caractéristiques, il faudrait insister sur la longueur des séries. On note des variations sensibles suivant que l'on prend une décennic ou une autre; d'après les spécialistes de la météorologie en Tunisic, on pourrait y voir l'influence des cycles solaires.
M. BEDIOT. - Je suis toujours un peu surpris - et ce n'est pas spécial à votre exposé, mais d'unc manière générale que l'on pose a posteriori le choix des lois statistiques.

Mon impression est qu'on applique un certain nombre de "recettes de cuisine" sans chercher à essayer à les relier aux phénomènes physiques.

En outre, les écarts entre certains ajustements sont faibles (quelques $\mathrm{mm} / \mathrm{h}$ ). Or, on sait l'imprécision des mesures qui peut exister à la suite d'erreurs qui interviennent en cascade. Vous avez parlé de capteurs à siphon et à flotteur; il y a aussi les augets basculeurs. Il y a, ensuite, toutes les techniques de dépouillement manuel qui sont la cause de nombreuses incertitudes.

J'ai la conviction que l'étencue des erreurs peut très bien couvrir l'étendue des écarts entre les différents ajustements.

M. THIRRIOT. - Je vous remercie de votre remarque. Je dois avouer que je me suis résigné à utiliser les formules de Montana parce que les responsables tunisiens voulaient profiter de l'expérience réglementaire française que constitue l'Instruction Technique. Entre les variations des paramètres $a$ et $b$ de la formule de Montana il peut y avoir une compensation qui cache un peu la nature profonde des choses.

M. DERI. - Tout d'abord, je voudrais féliciter M. THIRRIOT pour les travaux qu'il a dirigés en vue de rechercher des lois régionales.

Ma question concerne une remarque faite par M. THIRRIOT. Si j'ai bien compris, vous avez dit que vous n'aviez pas trouvé une correspondance entre l'intensité de la pluie et la pluviométrie interannuelle. Puis-je demander quelques renseignements supplémentaires sur cette remarque, parce qu'il y a des expériences qui prouvent qu'il y a une certaine correspondance entre l'intensité de la pluie de courte durée et la pluviométrie interannuelle. A titre de similitude, je citerai le gradex de MM. GUILLOT et DUBAND, où la pluviométrie interannuelle est plus faible dans le cas où le gradex est plus fort et inversement.

Est-ce que les stations de pluviométrie de Tunisie sont assez représentatives pour tirer une conclusion selon laquelle il n'y a pas une correspondance acceptable à ce niveau ?

Je lo repète, il s'agit d'un excellent travail pour élargir l'expérience en pluviométrie et en loi d'application de la pluie de courte durće.

M. THIRRIOT. - Je vous remercie de vos appréciations. Peut-être M. GUILlơ pourra t-il donner son point de vue sur votre question concernant la méthode du gradex?

Pour ce qui concerne la relation entre lois des averses et pluviométrie interannuelle, je montrerai à nouveau les résultats concernant Bizerte et Gabès. La différence des moyennes annuelles est énorme et pourtant les lois des averses sont bien proches. 
M. GUISCAFRE. - Il semble que l'on ait fait abstraction de l'appareillage qui a fourni les enregistrements utilisés lors de cette étude. Or, ayant nous-mêmes utilisé pour des études similaires en Tunisie, mais plus ponctuelles, une partie des enregistrements ayant servi à celle-ci, nous avons dû écarter de nos échantillons un nombre important d'années-station, particulièrement celles des stations synoptiques de la Météorologie Nationale Tunisienne, qui a utilisé pour un certain nombre de ces postes des pluviographes Précis-Mécanique équipés d'une bague réceptrice de $2000 \mathrm{~cm}^{2}$, brute de fabrication. Or, sans réalésage de l'ajutage, les entonnoirs correspondants se mettent en charge lors d'intensités voisines de $60 \mathrm{~mm} / \mathrm{h}$. Ce qui fausse bien sûr l'échantillonnage pour les études d'intensité-durée. Parmi les postes synoptiques incriminés sont inclus, entre autres, ceux de Bizerte et de Gabès. Ces anomalies ont été, par ailleurs, signalées à la Météorologie Nationale Tunisienne.

Le Président. - C'est une remarque importante, qui ne concerne d'ailleurs pas que la Tunisie.

$\mathrm{Si} \mathrm{M}$. GUILLOT le veut bien, je souhaiterais qu'il fasse $\mathrm{sa}$ communication maintenant, ce qui me paraît un ordre plus logique. De plus, elle ferait la transition avec l'analyse des épisodes pluvieux que nous allons examiner par la suite. 